\title{
Understanding Genetic Diversity of the Liver Fluke Fasciola hepatica
}

Mine Dosay-Akbulut ${ }^{\mathrm{a}, \mathrm{b}, *}$, Alan Trudgett ${ }^{\mathrm{b}}$, and Michael Stanhope $\mathrm{e}^{\mathrm{b}, \mathrm{c}}$

a Afyon Kocatepe University, Veterinary Faculty, Medical Biology and Genetics

Department, Afyon, Turkey. Fax: 00902281349. E-mail: minedosay@hotmail.com

b Biology and Biochemistry, Quenn's University of Belfast, Belfast, UK

c Department of Population Medicine and Diagnostic Sciences, College of Veterinary

Medicine, Cornell University, Ithaca, NY, USA

* Author for correspondence and reprint requests

Z. Naturforsch. 60c, 774-778 (2005); received January 19/March 8, 2005

Economical breeding is important to obtain maximum gain from the breeding in the animal sector. The economic loss has to be eliminated or should be minimized. The liver fluke, Fasciola hepatica, present mostly in sheep and dairy cattle affect the yield of animals and even cause their death. To eliminate or minimize the impact of these parasites on the animals, it is important to understand the genetic diversity of the liver fluke populations and the relationship between parasite and host at regional bases. This research was carried out to determine diversity by sequence analysis of the mitochondrial ND1 gene and ribosomal ITS1 region.

Key words: Fluke, Mitochondrial DNA and Ribosomal ITS

\section{Introduction}

The liver fluke, Fasciola hepatica, is a major cause of economic loss to the stock- raising industry in temperate regions of the world. In parts of NW Europe infection rates of the liver may reach $60 \%$ of sheep and dairy cattle (Garey and Wolstenholme, 1989).

Although infections are rarely lethal in cattle (as it can be in sheep) it is associated with a reduction in milk yields, failure to gain weight, and poor quality meat. At present infections are controlled by periodic dosing of cattle and sheep with various drugs. Fasciolisis is a re-emerging parasitic disease that mostly effects the Fasciola hepatica and its most common snail host Limnae truncatula in the Bolivian Altiplano, which has been introduced from Europe (Meunier et al., 2001).

Liver flukes are polymorphic and vary morphologically depending upon the host. Specimens from different hosts manifest differences far greater than those usually employed to distinguish between species of other flatworms. A range of morphological types of Fasciola has been reported in southeast Asian countries as well as in South America. These morphological variants, as well as the different definitive and intermediate hosts the parasites inhabit, suggest the possibility that there may be a complex of subspecific or distinct evolutionary lineages within the currently classified species Fasciola hepatica.
Very little molecular population genetics or phylogeographic work has been conducted on Fasciola spp. The few studies, that have dealt with population differences in isozymes, suggest that proteins are unlikely to provide the level of resolution necessary to detect cryptic species or distinct evolutionary lineages. Even less work has been conducted on nucleic acid variation in liver flukes and there are no published accounts of molecular phylogeography studies involving DNA data.

Little is known about genetic diversity and its distribution within the parasite genus Fasciola. Genetic diversity in the liver fluke might be associated with resistance to flukicidal drugs or manifest itself in different host-parasite immune responses. Either of these possibilities would have important implications for the design of therapeutic strategies.

After eggs are passed to the outside with definitive host faeces, a miracidium larvae hatch from the egg and enter a snail (asexual reproduction) developing to a cercaria larvae. Cercaria are released and encyst on vegetation, known as metacercaria, which is ingested by the host. Sexual reproduction occurs within the host, possibly hermaphroditically.

The principal aim of this study is to provide a thorough understanding of liver fluke genetic diversity, as well as the distribution of that genetic diversity, both in a phylogenetic and geographic context. 


\section{Materials and Methods}

Ribosomal ITS (internal transcribed spacer) was chosen because it is known to evolve relatively rapidly, and the mt DNA ND1 gene in order to provide a mtDNA comparison to the nuclear ITS sequence, the ND1 gene and ribosomal ITS1 region were sequenced. ND1 was chosen because its sequence is available, and thus we can easily design PCR (polymerase chain reaction) primers to amplify these regions from our populations. Furthermore, ND1 is known to be quite variable

ITS1 primer positions: in a range of other taxa for which it has been examined. Complete sequences of mtDNA are currently available from two nematodes, a crustacean, two sea urchins (Echinoderm), two insects - Drosophila (fruit flies) and Apis (honey bee) -, a bird, frog, carp (fish), human, and several other mammals. The gene organisation is conserved in vertebrates but differs in several invertebrate groups. The gene organisation in flatworms is not known. In this study, we designed primers from the available sequence CO1 and ND1 gene of different species, chosen as closest to the platyhelminthes, and used them to amplify the aimed part.

\section{8-S3 F: 5' GTAGGTGAACCTGCGGAAGGATCATT $\quad$ 3' ITS1 \\ 5.8-S4 R: 5' TTCATCGATCCACGAGCCGAGTGAT $\quad 3^{\prime} \quad$ ITS1 \\ 5.8-S5 R: 5' GGTGTTCATGTGTCCTGCAGTTCACATT $3^{\prime} \quad$ ITS1}

ND1 primer positions:

$\begin{array}{rlll}\text { 1F: 5' } & \text { TTATTTGGG TTTAAGTAGGTT } & 3^{\prime} & \text { ND1 } \\ 790 \mathrm{~F}: & \text { 5' ACCGTACTCCATTGGATTATGCT } & 3^{\prime} & \text { ND1 } \\ 997 \mathrm{R}: & 5 ' \text { TGCTACTTTACCTCGTGTTCGGTAT } & 3^{\prime} & \text { ND1 } \\ \text { 3R: 5' TATGTGGCGTTATGCTCTTTTATT } & 3^{\prime} & \text { ND1 }\end{array}$

The present source of genetic data is based on DNA sequences derived from the mtDNA ND1 gene, as well as the ITS1 region of the ribosomal cistron. Both loci were solated using PCR. These loci were chosen because of their high levels of variation in a wide range of other taxonomic groups. Primers for ITS1 were based on conserved regions at the $3^{\prime}$ end of the $18-S$ gene and within the 5.8-S locus. Primers for the ND1 gene were based on the published sequence for this locus from F. hepatica (Garey and Wolstenholme, 1989); the sequence reported in this preliminary report starts at the $3^{\prime}$ end of the gene. DNA sequences were obtained via direct sequencing methods, employing dye terminator cycle sequencing reactions that were subsequently loaded on an ABI automated DNA sequencer.

All liver flukes sampled in this preliminary report came from sheep and cattle hosts in Northern Ireland. For the ND1 gene these sequences were also compared to the single available North American sequence for $F$. hepatica from cattle. All DNA extractions were conducted on single liver flukes, using proteinase $\mathrm{K}$ and $70 \%$ phenol/water/chloroform. For ITS1, sequences were obtained for three liver flukes from each of two cattle and two sheep hosts, for a total of 12 sequences. For ND1, a total of 11 sequences were obtained, including 3 flukes from each of two sheep, and four flukes from two cows, 3 from a single cow and 1 from another one which are all collected from Northern Ireland; these sequences came from the same liver flukes that were sampled for ITS1. Apart from these, one cattle liver fluke was collected from North America (Utah) and used as a single-sequence in phylogenetic analysis for the ND1 gene only.

A Perkin Elmer DNA Thermal Cycler 480 or a PTC-100TM Programmable Thermal Controller (MJ Research, Inc, Waltham, USA) was used for all PCR amplifications. After extraction of tissue sample, the double-stranded genomic DNA was used in 30 cycles of PCR amplification: 5 min initial denaturing at $94{ }^{\circ} \mathrm{C}$, followed by $1 \mathrm{~min}$ at $94{ }^{\circ} \mathrm{C}$ and either $1 \mathrm{~min}$ annealing at $50-55^{\circ} \mathrm{C}$, followed by 2 min extension at $65^{\circ} \mathrm{C}$ or $3 \mathrm{~min}$ annealing plus extension at $60-65^{\circ} \mathrm{C}$, after 30 cycle, in a final 10 min extension at $65^{\circ} \mathrm{C} .50 \mu \mathrm{l}$ reaction mixture contained $200 \mathrm{ng} \mu \mathrm{l}^{-1}$ of two external primers, $10 \mathrm{~mm}$ of each nucleotide (dATP, dCTP, dGTP and dTTP) ultrapure dNTP set from Pharmacia Biotech (Denver, USA), $17.5 \mathrm{~mm} \mathrm{MgCl}_{2}$ and $1.75 \mathrm{U}$ of Taq polymerase (Boehringer Mannheim, Germany) or Taq polymerase (Expand ${ }^{\mathrm{TM}}$ High Fidelity PCR system, Boehringer Mannheim) and at least 300 ng genomic DNA. After the first PCR to estimate the size of the amplified fragment, this 
PCR product was run out on a $0.7 \%$ agarose gel, stained with ethidium bromide, visualised under low intensity ultraviolet light and photographed. After determination of the approx. size of this fragment, the PCR sample was run on a $1 \%$ low melt agarose gel. Then the band was visualised under low intensity ultraviolet light, excised and melted in a $65^{\circ} \mathrm{C}$ heating block. This excised amplified band was amplified again with the same PCR technique by using the same or internal primers. The final fragments were purified for sequence by using a high- pure PCR product purification kit, according to the manufacturer's instruction.

The cycle sequence reaction was carried out using either a Perkin Elmer DNA Thermal Cycler 480 or a PTC-100TM Programmable Thermal Controller (MJ Research, Inc.). The PCR amplified products were sequenced directly by using the dideoxy chain termination method. $20 \mu \mathrm{l}$ dye-termination reaction contained $300-500 \mathrm{ng}$ of purified PCR amplified product, $5 \mathrm{pmol}$ of each primer and $20 \mathrm{~mm} \mathrm{MnSO}_{4}$. The cycle included first a denaturation at $96^{\circ} \mathrm{C}$ for $1 \mathrm{~min}$, followed by 25 cycles of $50 \mathrm{~s}$ at $96^{\circ} \mathrm{C}, 4 \mathrm{~min}$ at $62^{\circ} \mathrm{C}$, and $20 \mathrm{~s}$ at $50{ }^{\circ} \mathrm{C}$. After that, the reactions were loaded onto an ABI 373A automated sequencer.

Alignments were obtained by eye, and DNA sequences were analyzed using PHYLIP [a phylogeny program package by Felsenstein (1993)]. A total of 419 and $273 \mathrm{bp}$ of aligned sequences has been found for each of the ITS1 and ND1 loci, respectively. Our Genbank search showed similarities in ND1 gene sequence of Garey and Wolstenholme (1989) and Le et al. (2000), supporting our results. Our Genbank search indicated no ITS1 sequence for Fasciola hepatica. Two different analyzing methods, which are maximum parsimony and neighbor-joining, within the PHYLIP 3.5c (Felsenstein, 1993) were used to exclude a possible method effect. Sequence divergence was calculated for both loci using the Jukes and Cantor (1969) method, and neighbor-joining trees were constructed from the resulting matrices. Maximum parsimony trees were determined by randomizing the input order 20 times, using the jumble option available within PHYLIP. For both the ND1 and the ITS1 loci, significance tests were performed between user-defined or constrained trees and the maximum parsimony tree, designed to assess the likelihood of a monophyletic grouping of "cattle liver flukes" or "sheep liver flukes".

\section{Results}

Jukes and Cantor (1969) distances for the ND1 gene ranged from 0.011 to 0.062 (approx. 1-6\% sequence divergence) and for the ITS1 gene from 0.010 to 0.068 (approx. $1-7 \%$ sequence divergence). Neighbour-joining trees constructed from these divergence matrices did not show a monophyletic grouping of sheep liver or of cattle liver flukes (Figs. 1 and 2). Furthermore, the ND1 sequence of the North American cattle liver fluke was not anymore divergent from either cattle or sheep liver flukes from Northern Ireland, with sequence divergence figures involving this North American fluke ranging from 0.011 to 0.042 .

Parsimony analyses of both genes agreed with the neighbour-joining trees in not supporting a monophyletic grouping of cattle or sheep liver flukes. In both cases the most parsimonious trees had mixed groupings of cattle and sheep flukes (Figs. 3, 4). Constraining the data to support a mo-

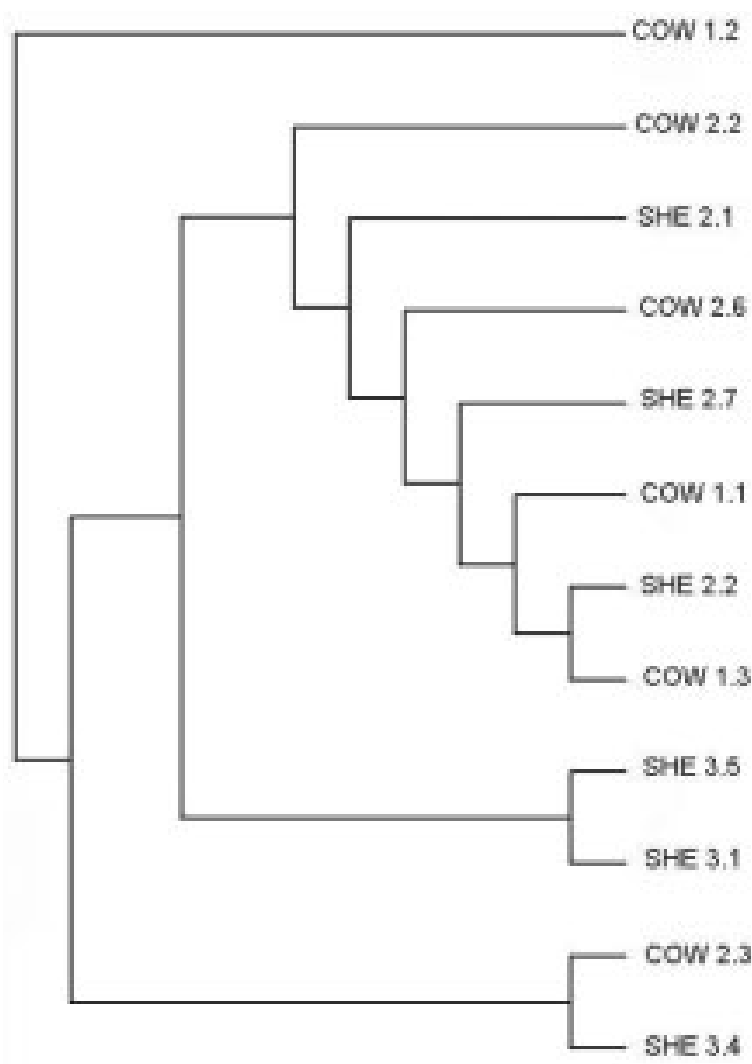

Fig. 1. An unrooted maximum parsimony tree for ITS1 sequence. 


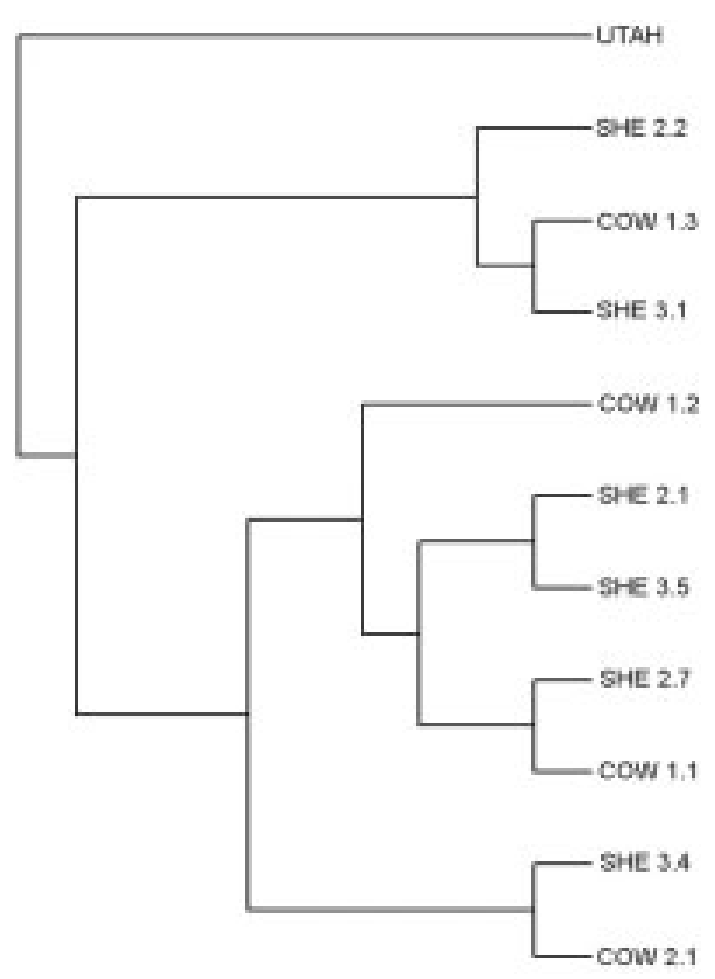

Fig. 2. An unrooted maximum parsimony tree for ND1 sequence.

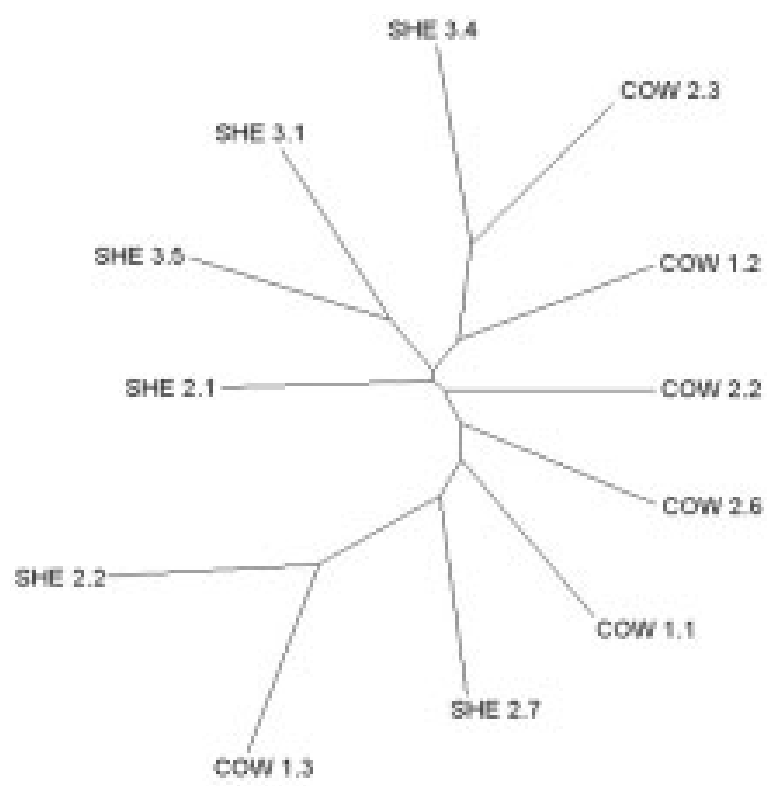

Fig. 3. An unrooted neighbor joining tree for ITS1 sequences divergence.

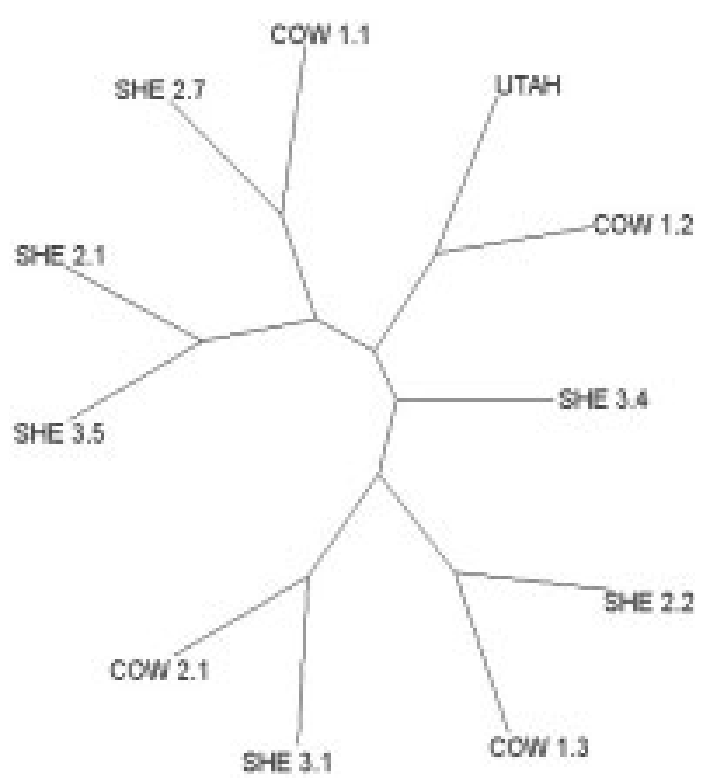

Fig. 4. An unrooted neighbor joining tree for ND1 sequence divergence.

nophyletic cattle and sheep liver fluke clade added seven additional substitutions to the most parsimonious tree for the ND1 gene, representing an $18 \%$ increase to the score of this most parsimonious tree. A similar analysis involving ITS1 also added +7 , representing in this case a $10 \%$ increase in score. For both genes and analyses these trees, supporting a monophyletic grouping of cattle or sheep flukes, were judged to be significantly different from the most parsimonious trees.

Maximum parsimony analysis of ND1 gene placed sheep 2.7 and cow 1.1 together. As well as cow 2.1 and sheep 3.4, and sheep 3.1 and cow 1.3 placing together, indicated that the liver fluke samples, collected from different hosts, can be placed together. The liver fluke sample collected from Utah (North America) was placed outside of all other samples, indicating that this sample had big differences from the others.

ITS1 maximum parsimony analysis results placed cow 2.3 and sheep 3.4, and cow 1.3 and sheep 2.2 together, although only 12 sequences for ITS1 and 11 sequences for ND1 were used. Although this is still a preliminary research, the results support the finding that different samples, collected from the different hosts, can be placed together and there is no difference in their evolution. 


\section{Discussion}

The Genbank search shows some studies containing Fasciola hepatica and its molecular evolution, collected from different host and different geographic areas. Some of them are summarized below, to support our results with a limited number of samples.

The polymorphism on ND1 and CO1 mitochondrial genes was detected first on four liver flukes, collected from Ukraine, Belarus, Moscow and Mordovian regions by Morozova et al. (2004). In total 292 bp sequences for ND1 and 433 bp sequences for CO1 were collected and 2.7 and $0.9 \%$ of total sequence polymorphism accounted. Seven haplotypes were detected within four samples. The results support the assumption that there are genetic differences between four geographically distant populations of Fasciola hepatica (Morozova et al., 2004).

In another study by Morozova and coworkers (2002) the method of random DNA amplification by PCR was applied to estimate variation in two trematode species, Fasciola hepatica and Dicrocoelium dendriticum. The results indicate that there was a 35.5 to $83.2 \%$ polymorphism level in the Fasciola hepatica population and genetic similarity was found higher between five Fasciola hepatica samples being $41.4 \%$, and they formed two unequal clusters. The estimation of intraspecific variation in total Fasciola hepatica samples indicate the absence of a clear genetic differentiation between the parasites samples collected from different host individuals (Morozova et al., 2002).

Felsenstein J. (1993), PHYLIP: Phylogeny Inference Package. Version 3.5c. Distributed by John Felsenstein, University of Washington, Seattle, USA.

Garey J. R. and Wolstenholme D. R. (1989), Platyhelminth mitochondrial DNA: Evidence for early evolutionary origin of a tRNAserAGN, that contains a dihydrouridine arm replacement loop, and of serinespecifying AGA and AGG codons. J. Mol. Evol. 28, 374-387.

Jukes T. H. and Cantor C. R. (1969), Evolution of Protein Molecules. In: Mammalian Protein Metabolism (Munro H. N., ed.). Academic Press, New York, pp. 21-32.

Le T. H., Blair D., Agatsuma T., Humair P. F., Campbell N. J., Iwagami M., Litlewood D. T., Peacock B., Johnston D. A., Bartley J., Rollinson D., Herniou E. A., Zarlenga D. S., and Macmanus D. P. (2000), Phylogenies inferred from mitochondrial gene orders a cautionary tale from the parasitic flatworms. Mol. Biol. Evol. 17, 1123-1125.
Geographical variation of the liver fluke, Clonorchis sinensis, from Korea and China based on the karyotypes, zymodeme and DNA sequences analyses indicates that three populations of Clonorchis sinensis show high homogeneity in the nucleotide sequences of the 18-S rDNA, ITS2 and mtCO1 gene (Park and Yong, 2001).

These data should be regarded as preliminary, since they do not include higher number of samples and we know that more samples give more reliable results. We suggest that the flukes inhabiting cattle and sheep are no distinct evolutionary entities. If the cattle and sheep flukes were clearly distinct taxa at the molecular genetic level, then they should form monophyletic assemblages, and they do not with either the ITS1 or ND1 sequences. One source of difficulty in using sequence data to assess, whether different groups represent distinct evolutionary entities, lies in the level of sequence difference. If the sequences are highly similar or nearly identical, then there are few or no phylogenetically informative positions to evaluate similarities and/or differences. Apparently this is not the case with these loci, at the moment showing 20 phylogenetically informative positions over a stretch of $273 \mathrm{bp}$ of the ND1 gene, and 25 informative positions for the ITS1 locus.

Although at present only a single sequence from North America is available for comparison, it shows no more sequence difference than Northern Ireland flukes, suggesting the possibility that liver flukes all over the world are relatively undifferentiated.

Meunier C., Tirard C., Hurtrez-Bousses S., Durand P., Bargues M. D., Mascoma S., Pointier J. P., Jourdane J., and Renaud F. (2001), Lack of molluscan host diversity and the transmission of an emerging parasitic disease in Bolivia. Mol. Ecol. 10, 1333-1340.

Morozova E. V., Ryskov A. P., and Semyenova S. K. (2002), RAPD variation in two trematode species (Fasciola hepatica and Dicrocoelium dendriticum) from a single cattle population. Genetika 38, 11551162

Morozova E. V., Chrisanfova G. G., Arkhipov I. A., and Semyenova S. K. (2004), Polymorphism of the ND1 and $\mathrm{CO} 1$ mitochondrial genes in populations of liver fluke Fasciola hepatica. Russ. J. Genet. 40, 817-820.

Park G. M. and Yong T. S. (2001), Geographical variation of the liver fluke, Clonorchis sinensis, from Korea and China based on the karyotypes, zymodeme and DNA sequences. Southeast Asian J. Trop. Med. Public Health 32, Suppl. 2, 12-16 